\title{
Teeth Extractions in Subjects Undergoing Radiotherapy for Head and Neck Cancers: A Systematic Review on the Clinical Protocols for Preventing Osteoradionecrosis (ORN). Extractions before Radiotherapy (Part 1) ${ }^{\dagger}$
}

\author{
Cosimo Rupe 1,*, Gioele Gioco ${ }^{1}$, Giuseppe Troiano ${ }^{2}$, Michele Giuliani ${ }^{2}$, Maria Contaldo ${ }^{3}$ and \\ Carlo Lajolo ${ }^{1}$ \\ 1 Head and Neck Department, “Fondazione Policlinico Universitario A. Gemelli-IRCCS”, School of \\ Dentistry, Università Cattolica del Sacro Cuore, 00168 Rome, Italy; gioele.gioco@hotmail.it (G.G.); \\ carlo.lajolo@unicatt.it (C.L.) \\ 2 Department of Clinical and Experimental Medicine, University of Foggia, 71121 Foggia, Italy; \\ giuseppe.troiano@unifg.it (G.T.); michele.giuliani@unifg.it (M.G.) \\ 3 Department of Medical-Surgical and Odontostomatological Specialties, University of Campania “Luigi \\ Vanvitelli", 80138 Naples, Italy; maria.contaldo@gmail.com \\ * Correspondence: cosimorupe@gmail.com; Tel.: +39-3929381949 \\ + Presented at the XV National and III International Congress of the Italian Society of Oral Pathology and \\ Medicine (SIPMO), Bari, Italy, 17-19 October 2019.
}

Published: 10 December 2019

Osteoradionecrosis (ORN) of the jaws is the most severe side effect, in some case life-threatening, of radiotherapy for head and neck cancer [1]. Tooth extractions before radiotherapy seems to lead to a reduction of ORN onset, nevertheless the real ORN rate and its risk factors are still unclear [2]. The aim of this systematic review is to determine the rate of ORN due to tooth extraction before the beginning of RT in patients affected by head and neck cancer and to identify any possible risk factor for ORN onset.

PRISMA protocol was used to evaluate and present the results. PubMed, Scopus, and Web of Science were used as search engines: only English full-length papers of clinical trial and observational studies both prospective and retrospective, published in peer-reviewed journals, were investigated. Inclusion criteria: minimum sample size 10 patients who underwent extraction before radiotherapy in head and neck district; minimum 6 months of follow-up after RT; ORN diagnosis criteria clearly defined in the text; specified if ORN developed at extraction site or not. Cumulative meta-analysis was performed calculating the pooled proportion (PP) of ORN occurrence rate. Meta-analysis was performed at random effects model with the Der-Simonian Liard method. All the statistical analyses were performed with the software Open Meta-Analyst version 10. PROSPERO registration code is CRD42018079986.

Among 2020 records screened, only 8 were included in this review (Figure 1). Sixteen of 494 patients who underwent tooth extraction before radiotherapy developed ORN at extraction site, with an ORN incidence of 2.2\% (95\% Confidence of Interval $=0.8-3.9, p<0.204, \mathrm{I}^{2}=2695 \%$ ) (Figure 2). All cases of ORN due to pre-RT dental extraction were reported in mandible. 


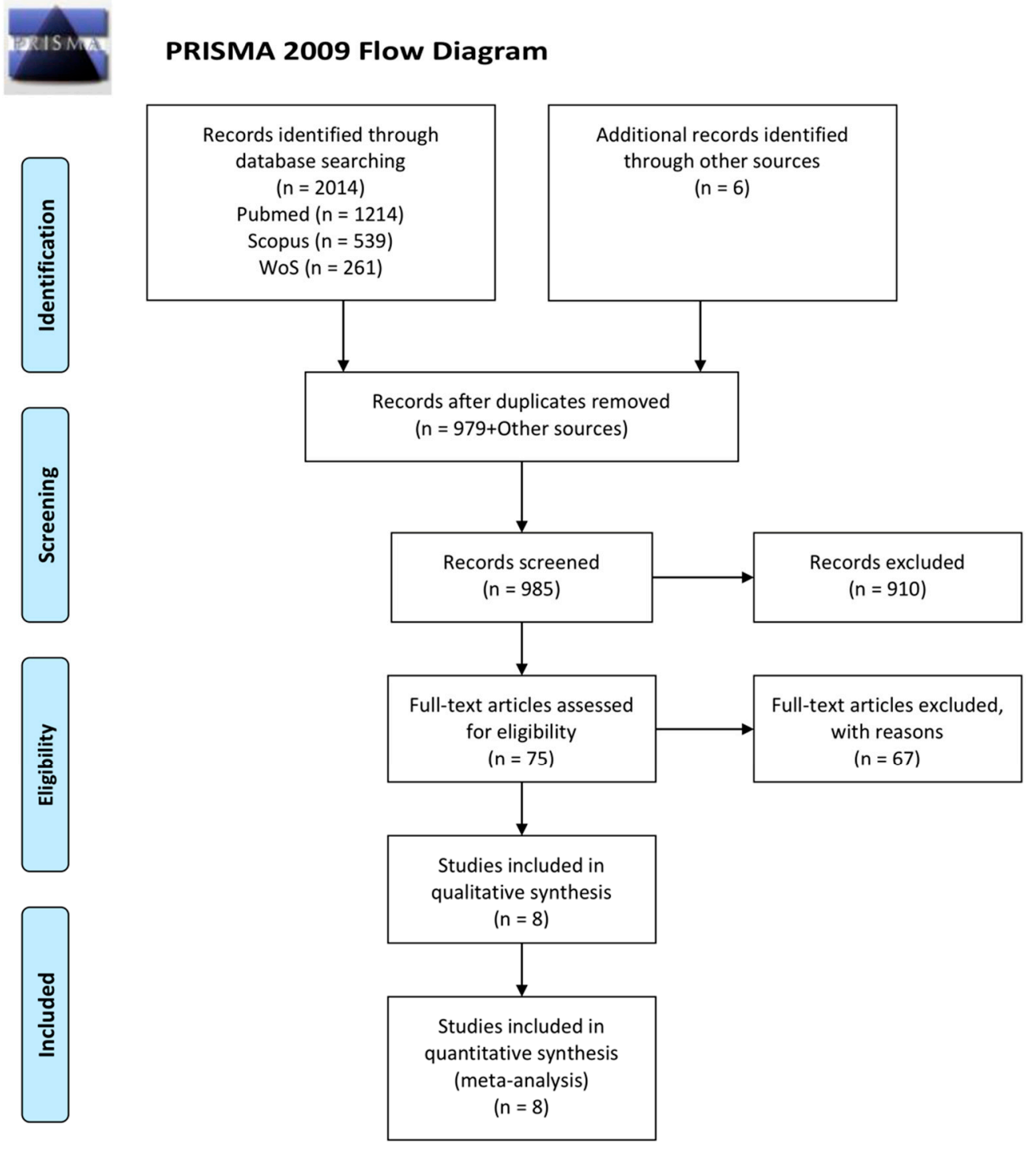

Figure 1. PRISMA flow-chart of the inclusion process.

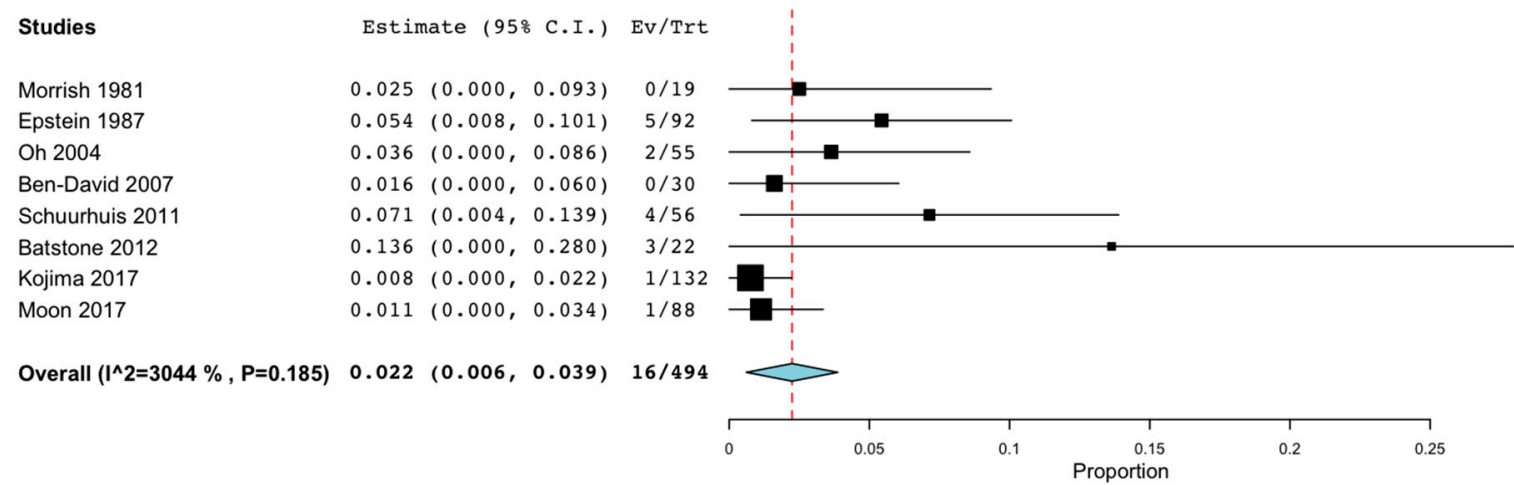

Figure 2. Pooled data and box plot stratified for studies.

The considerable amount of missing data didn't allow us to identify other possible risk factor for ORN onset (i.e., surgical protocol, time interval between the extraction and the beginning of radiotherapy, dose). Major efforts should be done to perform sounder methodological clinical investigations. It is general recommendation that patients should undergo a dental examination and oral foci should be removed before the beginning of radiotherapy for head and neck cancer, in order 
to reduce the risk of occurrence of undesirable effects. Although tooth extraction prior to radiotherapy leads to a reduction of ORN incidence, this systematic review shows that ORN risk is still high and special attention should be posed to extraction site.

Conflicts of Interest: The authors declare no conflict of interest.

\section{References}

1. Marx, R.; Johnson, R. Studies in the radiobiology of osteoradionecrosis and their clinical significance. Oral Surg. Oral Med. Oral Pathol. 1987, 64, 379-390.

2. Chronopoulos, A.; Zarra, T.; Ehrenfeld, M.; Otto, S. Osteoradionecrosis of the jaws: Definition, epidemiology, staging and clinical and radiological findings. A concise review. Int. Dent. J. 2018, 68, 22-30.

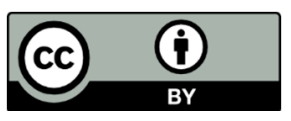

(C) 2019 by the authors. Licensee MDPI, Basel, Switzerland. This article is an open access article distributed under the terms and conditions of the Creative Commons Attribution (CC BY) license (http://creativecommons.org/licenses/by/4.0/). 